\title{
Can intervals in extracorporeal shock wave lithotripsy sessions affect success in the treatment of upper ureteral stones?
}

\author{
Turgay Turan, Ozgur Efiloglu, Yavuz Onur Danacioglu, Furkan Sendogan, Meftun Culpan, Bilal Gunaydin, \\ Ramazan Gokhan Atis, Turhan Caskurlu, Asif Yildirim \\ Department of Urology, Faculty of Medicine, Istanbul Medeniyet University, Istanbul, Turkey
}

Videosurgery Miniinv 2018; 13 (4): 507-511 DOI: https://doi.org/10.5114/wiitm.2018.75873

\begin{abstract}
Introduction: Managing upper ureteral stones with shock wave lithotripsy (SWL) has been done for many years. However, no conclusive data have been found on the intervals required between repeated SWL sessions.

Aim: To identify the most optimal intervals between SWL sessions.

Material and methods: Between September 2015 and December 2016, 80 patients undergoing elective outpatient lithotripsy who required repeated SWL sessions for ureteral stones were evaluated. These patients were categorised into two groups according to different SWL session intervals. The intervals between SWL sessions $(n=40)$ for the first group lasted one day, whereas those for the second group $(n=40)$ lasted 1 week. The data recorded during SWL included visual analogue scale (VAS) scores, total number of shocks, total energy used and number of sessions. The stone clearance rates, number of urinary tract infections, complication rates and VAS scores were compared between the two groups.

Results: All patients' clinical outcomes after SWL therapy are shown in Table I. The stone clearance rate for group 1 was $70 \%$, whereas that for group 2 was $72.5 \%(p=0.805)$. Additionally, the number of urinary tract infections, complications and VAS scores were similar for both groups. In group 1, 3 patients had steinstrasse, two suffered from severe renal colic, and one had a urinary tract infection. In group 2, 3 patients endured steinstrasse, and 2 patients experienced severe renal colic during the study.

Conclusions: Short-interval SWL sessions are safe and effective for treating upper ureteral stones, and such sessions do not increase complication rates.
\end{abstract}

Key words: shock wave lithotripsy, upper ureteral stones, repeat sessions.

\section{Introduction}

Extracorporeal shock wave lithotripsy (SWL) has been used for many years in the treatment of kidney stones. Up-to-date guidelines refer to SWL as a reliable replacement treatment for ureteroscopy (URS) in managing upper ureteral calculi smaller than 10 $\mathrm{mm}$ [1]. Shock wave lithotripsy is preferred for its applicability to outpatients, lack of anaesthesia and low risk of complications [2]. Ureteroscopy has been increasingly favoured for the treatment of upper ureteral stones because of its high success rate within a short period of time, which is facilitated by the use of miniature surgical instruments, increased imaging quality and the effective use of laser technology, all of which are parallel to the advancing technology in the field [3]. Well-known evidence shows the potential risk of renal injury after repeated treatment of kidney stones with SWL [4], but no evidence of a similar risk for ureteral stones exists because of

\section{Address for correspondence}

Dr. Turgay Turan, Department of Urology, Faculty of Medicine, Istanbul Medeniyet University, 34722 Istanbul, Turkey,

phone: +90 2165700000, e-mail: turanturgay@hotmail.com 
the lack of involvement of renal parenchyma in the shock wave exposure.

The best procedure for determining SWL session intervals for upper ureteral stones remains debatable, but clinical experience has indicated that lithotripsy could be performed with one-day intervals [1].

\section{Aim}

This study seeks to assess the efficiency and safety of SWL sessions with short intervals in the treatment of upper ureteral stones.

\section{Material and methods}

After approval from the ethics committee at Istanbul Medeniyet University was obtained, patients with upper ureteral stones (below the ureteropelvic junction, above the sacroiliac joints) diagnosed via non-contrast computed tomography between October 2015 and December 2016 and who received active treatment after a 2-week conservative treatment were enrolled in the study. All sessions were performed by technicians with a decade of experience in using SWL and were monitored by the same physician (TT) using a Siemens Modularis Variostar lithotripter (Siemens AG, Medical Solutions, Erlangen, Germany).

Urine culture, kidney function and coagulation tests were completed for each patient before the procedure. Patients with contraindications to SWL (pregnancy, coagulopathy, renal insufficiency, urinary tract infection or distal obstruction) were excluded from the study. No patients were deprived of nutrients or given prophylactic antibiotics before the procedure. Thirty minutes before the operation, $75 \mathrm{mg}$ of diclofenac sodium was injected into each patient intramuscularly. The patients were called in for follow-up on the second day of the first session to evaluate their need for additional sessions. Those who experienced stone fragmentation during SWL and the continuous spontaneous passage of these fragments after the first SWL session follow-up were not included in this study to avoid risking overtreatment. Those whose first sessions were unsuccessful and who were scheduled for a second session were divided into two groups at random. The first group of patients had their second session the following day after the first session, whereas the second group's second session was conducted after a week. If necessary, a third session took place at similar intervals.
Each session involved either 3,000 shocks or enough shocks to thoroughly fragment the stone. The delivery rate of the shock waves (SW) was 90 pulses per minute. The SW voltage began with a series of $500 \mathrm{SW}$ at $0.1 \mathrm{kV}$, which increased in $0.2-0.3 \mathrm{kV}$ steps at every $300 \mathrm{SW}$ to a maximum of $2.5-3.0 \mathrm{kV}$. We identified the real positions of the stones and observed continuity in fragmentation via fluoroscopy by using shot imaging at intervals of 300-500 shocks.

Stone localisation was checked with fluoroscopy, whereas ultrasound technology was used to evaluate renal damage and hydronephrosis. X-ray doses were not standard, as they were automatically calculated by fluoroscopy according to patient-specific parameters (age, height, weight and body mass index (BMI)). Routine urine culture, X-rays and ultrasonography (USG) were used to determine a procedure's success rate and complications before the repeated session. The pain scores for every session were evaluated by the patient using the VAS. Shock wave lithotripsy was considered successful if no stones were found in the X-rays and USG 2 weeks after the final session. Both groups were compared in terms of clinical characteristics, therapy success, complications and VAS.

\section{Statistical analysis}

Statistical analysis was performed using IBM SPSS Statistics version 21 (IBM Co., USA). Student's $t$-test and the $\chi^{2}$ test were used to compare the parameters of each group, and $p<0.05$ were considered statistically significant.

\section{Results}

This study included 54 male and 26 female patients with a mean age of 44.3 years who were diagnosed with upper ureteral stones and had an unsuccessful initial SWL session. Both groups shared similar mean ages and BMI $(p>0.05)$ (Table I). The mean stone size was $10.23 \pm 2.87 \mathrm{~mm}$ in group 1 and $10.2 \pm 2.82 \mathrm{~mm}$ in group 2, whereas the mean Hounsfield density in Hounsfield units (HU) of the stones was $869.16 \pm 266.6 \mathrm{HU}$ in group 1 and $869.3 \pm 251.1 \mathrm{HU}$ in group 2. Patients assessed their post-procedural pain in each session by using the VAS, the results of which were similar for both groups $(p>0.05)$ (Table I). All patients in both groups received at least two SWL sessions. Nineteen patients in group 1 and 16 in group 2 received three 
Table I. Clinical outcomes of shock wave lithotripsy therapy

\begin{tabular}{|c|c|c|c|}
\hline Parameter & Group $1(n=40)$ & Group $2(n=40)$ & $P$-value \\
\hline Age, mean \pm SD [years] & $41.4 \pm 12.9$ & $44.7 \pm 16.3$ & 0.394 \\
\hline M/F ratio & $28 / 12$ & $26 / 14$ & 0.613 \\
\hline $\mathrm{BMI}\left[\mathrm{kg} / \mathrm{m}^{2}\right]$ & $27 \pm 3.5$ & $28.6 \pm 6.3$ & 0.234 \\
\hline Stone size $[\mathrm{mm}]$ & $10.2 \pm 2.82$ & $10.23 \pm 2.87$ & 0.964 \\
\hline Left/right side & $14 / 26$ & $28 / 12$ & 0.002 \\
\hline Hounsfield units, mean \pm SD & $869.16 \pm 266.3$ & $869.3 \pm 251.1$ & 0.998 \\
\hline Average total energy [U] & $92.2 \pm 24.4$ & $89.97 \pm 33.4$ & 0.496 \\
\hline VAS ( $\left(1^{\text {st }}\right.$ session $)$ & $4.2 \pm 1.1$ & $4.2 \pm 1.2$ & 0.918 \\
\hline VAS ( $2^{\text {nd }}$ session $)$ & $4.23 \pm 1.1$ & $4.4 \pm 1.2$ & 0.599 \\
\hline Stone free rate ( $2^{\text {nd }}$ session), $n(\%)$ & $19(47.5)$ & $21(52.5)$ & 0.651 \\
\hline No. of patients (3rd session), $n(\%)$ & $19(47.5)$ & $16(32.5)$ & 0.485 \\
\hline VAS ( $3^{\text {rd }}$ session) & $4.27 \pm 1.6(n=19)$ & $4.6 \pm 1.4(n=16)$ & 0.601 \\
\hline Complications, $n(\%)$ & $6(15)$ & $5(12.5)$ & 0.688 \\
\hline Stone clearance, $n(\%)$ & $28(70)$ & $29(72.5)$ & 0.805 \\
\hline Fluoroscopy time [min] & $1.57 \pm 34$ & $1.54 \pm 29$ & 0.730 \\
\hline
\end{tabular}

SWL sessions ( $p=0.485$ ) with the same intervals. The stone-free rates of groups 1 and 2 after the second SWL session were $47.5 \%$ and $52.5 \%(p=0.651)$, respectively. The energy used during the procedure was similar in both groups $(p>0.05)$ (Table I).

The complications observed in group 1 included steinstrasse formation in 3 patients, urinary infection in 1 patient and severe renal colic in 2 patients. The complications in group 2 were severe renal colic in 2 patients and steinstrasse formation in 3 patients. One steinstrasse-afflicted patient from either group was treated conservatively, whereas endoscopic interventions were performed for patients who developed severe renal colic. The patient who developed a urinary infection was treated as an inpatient for 2 days, after which the patient received antibiotic therapy as an outpatient. The SWL success rates were similar in both groups: $28(70 \%)$ in group 1 and $29(72.5 \%)$ in group 2. All patients with failed SWL treatment were subsequently treated endoscopically.

\section{Discussion}

Both SWL and URS are commonly used to treat upper ureteral stones, and these treatments yield similar stone-free rates after 3 months. Shock wave lithotripsy therapy is involved in many retreatments and secondary procedures because of its lower hospitalization time, low complication rates, and fewer adjunctive procedures [2]. However, these treatments are inadequate for large ureteral stones that may require laparoscopic surgery [5]. Ureteroscopy is frequently preferred for its response speed during treatment because ureteral stones carry a greater risk of renal colic, ureteral obstruction, and renal dysfunction compared with kidney stones. Factors such as possible controlled stone fragmentation with laser technology, intervention through retrograde intrarenal surgery (RIRS), simultaneous flexible URS for pushback, controlled passage of fragments through the ureter, and high success rates in a short period are many reasons for choosing URS. That said, the current study demonstrates that SWL performed between short intervals results not only in swift success, but also in lower complication rates. An ideal goal is to eliminate the risks of general anaesthesia and surgery, by completing lithotripsy by keeping the ureteral passage open for a short while, such as 1 week. Even if short-interval SWL therapy fails, not much time would be required to advance to secondary treatment. 
The larger the size of a stone, the lower the likelihood of SWL's success [6]. Additionally, it is advised that SWL should only be performed on the few patients who have ureteral stones larger than $15 \mathrm{~mm}$ [7]. Harrogate et al. reported that during SWL, ureteral stones $(3.6 \mathrm{~mm})$ migrated less with respiration compared with kidney stones $(7.7 \mathrm{~mm})$ [8]. The main problem during SWL was the blockage of controlled stone fragmentation due to ureteric migration, thereby causing large stone fragmentations and even steinstrasse in patients with larger stone masses. There was no increase in the risk of steinstrasse after lithotripsy with short intervals when the stone sizes were similar in both groups in our study. Although conservative treatment is initially adequate if the patient is asymptomatic after steinstrasse, medical expulsive therapy (MET) could reduce the necessity of endoscopic therapy. Even if stone fragmentation is achieved after SWL, a waiting period is required for spontaneous passage; conservative procedures after SWL sessions, such as increased hydration and movement and the use of $\alpha$-blockers, could hasten colic attacks and the removal of stones [9].

There is a major risk of complications associated with bacteria which may be present in urine and/or the stone and be released during stone disintegration [10]. No prophylactic antibiotics were used during any procedures, and urine cultures - which came back as sterile - were taken before every procedure in this study. Whilst one patient in the short-interval SWL group had a urinary infection 7 days after the session, short intervals did not increase the risk of infection. Similarly, short-interval SWL did not affect the VAS scores. All patients who experienced no problems during the first session tolerated SWL with 1-day intervals.

Perinephric oedema, BMI, stone diameter, density, JJ stent, and delivery rates affect SWL success rates $[11,12]$. The clinical characteristics of the patients in our study were similar, and both groups received SWL with similar techniques by the same technician. Therefore, these factors are considered to have affected success in both groups to similar degrees. In a study by Yazici et al., increased $\mathrm{HU}$ adversely impacted success [13]; however, the HU values in our study were similar, and no patients had JJ stents prior to SWL.

In a study by Kilinc et al., URS was performed safely and successfully after SWL [14]. Patients whose stones are broken with SWL but do not achieve spontaneous passage or develop steinstrasse may have difficulty with endoscopic procedures due to ureteral stricture or stone-related mucosal injury and oedema. In our study, endoscopic procedures for patients who experienced unsuccessful SWL sessions were successfully completed.

This study has some major limitations. First, short-interval SWL sessions carry the risk of overtreatment. Second, no data are available on stone compositions. In this study, the reduction of SWL session intervals neither affected the success rate negatively nor caused further complications. SWL with short intervals can be performed for patients with upper ureteral stones to attain a stone-free state quickly. Sessions with short intervals can be chosen if no fragmented stones in the ureter are found after stone fragmentation is checked in the first follow-up. As early surgery has higher success rates, increases the quality of life and accelerates the return to daily life, SWL therapy with short intervals could be a good option.

\section{Conclusions}

Short-interval SWL sessions are safe and effective in treating upper ureteral stones. These sessions do not increase complication rates, so they are advisable as an active therapy for ureteral stones, especially if fast results are prioritised.

\section{Conflict of interest}

The authors declare no conflict of interest.

\section{References}

1. C. Türk (Chair) AP, Seitz C, Skolarikos A, et al. Guidelines Associates: S. Dabestani TD, Grivas N, Ruhayel Y. Eau Guidelines On Urolithiasis (Limited text update March 2017). 2017 [cited 14.12.2017]; Available from: https://uroweb.org/guideline/urolithiasis/?type=pocket-guidelines

2. Drake T, Grivas N, Dabestani S, et al. What are the benefits and harms of ureteroscopy compared with shock-wave lithotripsy in the treatment of upper ureteral stones? A systematic review. Eur Urol 2017; 72: 772-86.

3. Cui X, Ji F, Yan H, et al. Comparison between extracorporeal shock wave lithotripsy and ureteroscopic lithotripsy for treating large proximal ureteral stones: a meta-analysis. Urology 2015; 85: 748-56.

4. Hadj-Moussa M, Brown JA. Effect of high shock number on acute complication development after extracorporeal shockwave lithotripsy. J Endourol 2013; 27: 1015-9.

5. Kadyan B, Sabale V, Mane D, et al. Large proximal ureteral stones: ideal treatment modality? Urol Ann 2016; 8: 189-92. 
6. Niwa N, Matsumoto K, Miyahara M, et al. Simple and practical nomograms for predicting the stone-free rate after shock wave lithotripsy in patients with a solitary upper ureteral stone. World J Urol 2017; 35: 1455-61.

7. Aboutaleb H, Omar M, Salem S, Elshazly M. Management of upper ureteral stones exceeding $15 \mathrm{~mm}$ in diameter: shock wave lithotripsy versus semirigid ureteroscopy with holmium: yttrium-aluminum-garnet laser lithotripsy. SAGE Open Med 2016; 4: 2050312116685180.

8. Harrogate SR, Yick LS, Williams Jr JC, et al. Quantification of the range of motion of kidney and ureteral stones during shockwave lithotripsy in conscious patients. J Endourol 2016; 30: 406-10.

9. Skolarikos A, Grivas N, Kallidonis P, et al. The efficacy of med ical expulsive therapy (MET) in improving stone-free rate and stone expulsion time, after extracorporeal shock wave lithotripsy (SWL) for upper urinary stones: a systematic review and meta-analysis. Urology 2015; 86: 1057-64.

10. Chaussy CG, Tiselius HG. How can and should we optimize extracorporeal shockwave lithotripsy? Urolithiasis 2018; 46: 3-17.

11. Park HS, Gong MK, Yoon CY, et al. Computed tomography-based novel prediction model for the outcome of shockwave lithotripsy in proximal ureteral stones. J Endourol 2016; 30: 810-6.

12. Nguyen DP, Hnilicka S, Kiss B, et al. Optimization of extracorporeal shock wave lithotripsy delivery rates achieves excellent outcomes for ureteral stones: results of a prospective randomized trial. J Urol 2015; 194: 418-23.

13. Yazici O, Tuncer M, Sahin C, et al. Shock wave lithotripsy in ureteral stones: evaluation of patient and stone related predictive factors. Int Braz J Urol 2015; 41: 676-82.

14. Kilinc MF, Doluoglu OG, Karakan T, et al. Ureteroscopy in proximal ureteral stones after shock wave lithotripsy failure: is it safe and efficient or dangerous? Can Urol Assoc J 2015; 9 : E718-22.

Received: 12.03.2018, accepted: 12.04.2018. 